\title{
Evaluation quantitative des résidus de pyréthrinoïdes, d'aminophosphate et de triazines en zones de production de coton au Bénin par la méthode ELISA en phase liquide : cas des eaux de la rivière Agbado
}

\author{
Magloire Acakpo Nonvignon GBAGUIDI ${ }^{1 *}$, Henri Houénoukpo SOCLO ${ }^{1}$, \\ Yari Mohamed ISSA ${ }^{1}$, Benjamin FAYOMI ${ }^{2}$, Robert DOGNON ${ }^{1}$, Armelle AGAGBE ${ }^{1}$, \\ Clément BONOU ${ }^{3}$, Alassane YOUSSAO ${ }^{1}$, Léonce Firmin DOVONOU ${ }^{1,4}$ et \\ Ambaliou SANNI ${ }^{5}$ \\ ${ }^{1}$ Unité de Recherche en Ecotoxicologie et Etude de Qualité (U.R.E.E.Q)/Laboratoire de Recherche en Chimie \\ Appliquée (LERCA)/Ecole Polytechnique d'Abomey-Calavi (EPAC)/ Université \\ d'Abomey-Calavi (UAC) 01BP2009 Cotonou, République du Bénin. \\ ${ }^{2}$ Laboratoire de recherche en santé au travail et environnement/Institut des Sciences Biomédicales Appliquées \\ (ISBA)/Faculté ses Sciences de la Santé (FSS)/Université d'Abomey-Calavi (UAC), République du Bénin. \\ ${ }^{3}$ Laboratoire d'hydrobiologie Ecole Polytechnique d'Abomey-Calavi (EPAC)/Université d'Abomey-Calavi \\ (UAC) $01 B P 2009$ Cotonou, République du Bénin. \\ ${ }^{4}$ Laboratoire de Qualité de l'Eau de la Direction Générale de l'Eau (DGeau) du Ministère de l'Energie et de \\ l'Eau, République du Bénin.. \\ ${ }^{5}$ Laboratoire de Biochime et de Biologie Moléculaire, Faculté des Sciences et Techniques (FAST)/ Université \\ d'Abomey-Calavi (UAC) 04BP0320 Cotonou, République du Bénin. \\ *Auteur correspondant, E-mail :gbmag3@yahoo.fr
}

\section{RESUME}

Le présent travail réalisé au Bénin dans la commune de Savalou, a consisté en une recherche de résidus de pyréthrinoïdes, d'aminophosphates et de triazines dans l'eau et les sédiments de la rivière Agbado par la technique ELISA (Enzyme-Linked Immuno Sorbent Assay) en phase liquide. Quatre campagnes d'échantillonnage ont été menées dont deux en saison sèche et deux en saison pluvieuse. Au total quatre sites d'échantillonnage ont été explorés. Il s'agit de Mèdétèkpo, du Barrage de la Société des Eaux du Bénin, de la Station de traitement d'eau et du grand pont de Gobada. En saison sèche, aucun des résidus recherchés n'a été détecté dans l'eau. Dans les sédiments, les teneurs varient de 0,10 à 0,39 ppb pour le glyphosate et de 0,22 à $0,97 \mathrm{ppb}$ pour l'atrazine. En saison pluvieuse, dans l'eau, les taux varient de $0,05 \mathrm{ppb}$ à 0,475 ppb pour l'atrazine, de 0,10 à 1,316 ppb pour le glyphosate et de 0,75 à 4,450 ppb pour les pyréthrinoïdes. Dans les sédiments seul le glyphosate a été détecté jusqu'à un taux de 8,62 ppb. En moyenne ces valeurs sont supérieures aux normes de qualité de l'eau de boisson admises par l'Union Européenne. Face à ces niveaux de contamination, il importe que des mesures correctives soient prises.

(c) 2011 International Formulae Group. All rights reserved.

Mots clés : Contamination, eau, sédiments, Résidus de pesticides.

\section{INTRODUCTION}

L'usage en agriculture des produits phytosanitaires a permis d'améliorer les rendements et la diversité des cultures agricoles et de satisfaire la demande nutritionnelle liée à l'accroissement de la population (Scheyer, 2004). 
Cependant, cette utilisation a provoqué des effets néfastes directs et indirects sur l'environnement et sur l'homme (Katrijn et al., 2007). Des études ont montré la présence de résidus de pesticides à des teneurs atteignant $5900 \mathrm{ng} / \mathrm{L}$ d'atrazine et $3000 \mathrm{ppb}$ de pyréthrinoïde dans les eaux superficielles et souterraines en Grèce (Ioannis et al., 2006). Dans le sérum sanguin, les taux varient de 42,6 ppb pour le sulfate endosulfan à 24,8 et 21,9 ppb pour respectivement l'O,p'-DDT et le p,p'-DDT au Portugal (Celeste et al., 2006). Chez des fleuristes en Espagne, des teneurs de $2 \mu \mathrm{mol} / \mathrm{g}$ de créatine d'alkylphosphate ont été enregistrées (Marina et al., 2010). Dans les cultures maraîchères comme la carotte et le choux, des teneurs de 2,4 ppm à 9 ppm ont été retrouvées (Helena et al., 2004 ; Garey et al., 2010). Cette pollution due aux pesticides s'observe de façon plus aiguë en zone de production cotonnière dans les pays africains au sud du Sahara. Traoré et al. (2008) en Côte d'Ivoire à travers une étude menée sur le lait et les produits laitiers ont remarqué que les résultats obtenus révèlent une contamination générale du lait et du beurre par les pesticides organochlorés (POCs) avec des teneurs moyennes respectives de $42,75 \mu \mathrm{g} / \mathrm{kg}$ et $165,94 \mu \mathrm{g} / \mathrm{kg}$ pour l'hexachlorocyclohexane $(\mathrm{HCH}), \quad 31,58$ et $436,80 \mu \mathrm{g} / \mathrm{kg}$ pour l'endosulfan, de 8,70 et $59,91 \mu \mathrm{g} / \mathrm{kg}$ pour l'heptachlore et enfin 36,20 et $227,82 \mu \mathrm{g} / \mathrm{kg}$ pour le Dichlorodiphenyltrichloroethane (DDT). Au Bénin où le coton constitue la principale culture de rente marquée par d'intenses utilisations d'insecticides de synthèse (Banque Mondiale, 2004), la situation n'est pas aussi reluisante. SOCLO et al. (2003) ont montré que les eaux de surface en zones de culture de coton du Nord Bénin sont contaminées par des résidus de pesticides à des teneurs de $1790 \mathrm{ng} / \mathrm{L}$ de POCs totaux dans les rivières contre $530 \mathrm{ng} / \mathrm{l}$ dans les mares. Les études de Elisabeth et al. (2006a et 2006b), réalisées également sur le cour du fleuve Ouémé ont révélé non seulement les mêmes familles de molécules, mais à des concentrations plus élevées dans les sédiments et les tissus de poisson analysés. Si au Bénin les POCs ont fait l'objet de beaucoup de préoccupations à cause de leurs effets foudroyant et de leurs rémanences très élevées, les autres molécules de pesticides n'ont pas mobilisées tant d'attention. Or, parmi les pesticides utilisés en agriculture au Bénin et particulièrement dans la zone d'étude, les pyréthrinoïdes et les herbicides comme les aminophosphates et les triazines occupent une place de choix comme l'illustre le Tableau 1 qui présente les types et les quantités de pesticides utilisés officiellement en agriculture dans la commune de Savalou qui héberge la zone d'étude.

Dans le bassin versant de la rivière Agbado, objet de la présente étude, la plupart des riverains sont des cotonculteurs. La pression parasitaire sur le cotonnier couplée à l'analphabétisme des paysans amènent à un usage intensif et irrationnel des produits phytosanitaires soit directement en agriculture soit à des fins de pêche (Elisabeth et al., 2006a). Or en agriculture une bonne proportion des pesticides appliqués sur les parties aériennes des plantes, atteint directement le sol (Scheyer, 2004). Après les pluies cette fraction qui atteint le sol rejoint les eaux de surface par ruissellement superficiel ou les eaux souterraines par infiltration. La fraction de pesticide émise dans l'atmosphère par volatilisation finit aussi par regagner, sous forme de retombées atmosphériques, les sols et les eaux de surface lors des précipitations. Dans les plans d'eau qui sont le principal réceptacle de ces polluants dans l'environnement, les résidus de pesticides peuvent subir plus ou moins rapidement, selon leurs propriétés physicochimiques et les conditions du milieu, des réactions de dégradation physique et/ou biologique par hydrolyse, oxydation, réduction ou photolyse pour donner des sousproduits parfois plus toxiques que les produits parents. C'est le cas par exemple du parathion illustré par la Figure 1 qui, par oxydation sous l'action d'oxygénases, se transforme en paraoxon plus toxique que son précurseur (Blackburn et al., 2003). 
$\mathrm{Au}$ vu de ce qui précède, la recherche des résidus de pesticides de façon générale dans les écosystèmes aquatiques devient l'une des impératives à laquelle il faut satisfaire pour mieux éclairer des prises de décisions dans le contexte de développement durable, mais aussi celle des résidus de pyréthrinoïdes, d'aminophosphates et de triazines pour contribuer à l'évaluation du niveau de contamination des eaux de la rivière Agbado par les résidus de pesticides. Pour ce faire, la technique immunoenzymatique EnzymeLinked Immunosorbent Assay (ELISA) à été utilisée comme méthode analytique à cause de sa reproductibilité, de sa simplicité, de sa rapidité et de son coût relativement bas. De ce fait, elle peut servir dans les analyses de routines pour le suivi environnemental de la qualité chimique des eaux en matière de pollution par les pesticides.

\section{MATERIEL ET METHODES}

Milieu d'étude et sites de prélèvement des échantillons

La Figure 2 montre le bassin versant de la rivière Agbado. Il est localisé entre $7^{\circ} 35^{\prime}$ et $8^{\circ} 30^{\prime}$ latitude Nord et $1^{\circ} 50^{\prime}$ et $2^{\circ} 22^{\prime}$ longitude Est. La rivière prend sa source dans la Commune de Bantè, traverse la commune de Savalou qui abrite sa plus grande portion et se jette dans le fleuve Zou au niveau de la Commune de Djidja. D'une longueur d'environ $121 \mathrm{~km}$ et avec un bassin versant couvrant une aire estimée à $2635 \mathrm{~km}^{2}$, la rivière Agbado lessive un socle cristallin du précambrien qui ne facilite pas les forages souterrains. La population riveraine d'environ 100.835 habitants (INSAE, 2002), est essentiellement agricole et tire une bonne partie de ses besoins vitaux tels que les produits de pêche et l'eau d'alimentation de la rivière Agbado.

Le critère de choix de nos sites d'échantillonnage est basé sur le fait qu'ils sont tous des points d'approvisionnement en eau de boisson et soit un ancien ou récent point d'utilisation de pesticides à des fins de pêche ou agricole (sites 1, 2 et 4). Le Tableau 2 présente les différents sites d'échantillonnage avec leurs coordonnées géographiques et les périodes de prélèvement.

\section{Prélèvement d'échantillons}

Les échantillons d'eau sont prélevés dans des bouteilles ambrées préalablement nettoyées, séchées à l'étuve à $105{ }^{\circ} \mathrm{C}$ puis testées négatives par rapport aux résidus de pesticides recherchés. En effet les bouteilles ainsi traitées sont conditionnées avec $25 \mathrm{~mL}$ de méthanol. L'introduction préalable de ce solvant permet de limiter l'adsorption des résidus de pesticides à la paroi de la bouteille (Hock et al., 1994). $250 \mu \mathrm{L}$ de ce solvant sont prélevés après agitation pour être analysés en mélange équivolumique avec de l'eau milliQ.

$\mathrm{Au}$ niveau de chaque site d'échantillonnage un transect est réalisé dans le plan d'eau. Sur ce transect trois points sont échantillonnés, à savoir les abords des deux rives et le milieu du cours d'eau. L'eau est recueillie à $25 \mathrm{~cm}$ en dessous de la surface à l'aide de bouteilles lestées de 2 Litres. Les trois sous échantillons d'eau ainsi obtenus sont intimement mélangés en proportion égale pour en faire un échantillon composite dont 2 L sont transvasés dans une bouteille ambrée contenant $25 \mathrm{~mL}$ de méthanol.

Les échantillons de sédiments de surface $(0-5 \mathrm{~cm})$ sont prélevés à l'aide d'une benne Chippeck au niveau des trois points d'échantillonnage du transect. Ils sont ensuite mélangés en proportion égale puis emballés dans du papier aluminium préalablement nettoyé au pentane et à l'éthanol $95 \%$ et dont la propreté par rapport aux composés recherchés a été préalablement testée négative à l'instar des bouteilles de prélèvement d'eau par analyse du solvant de rinçage.

Les échantillons d'eau et de sédiments collectés et préservés contre la photodégradation, sont au fur et à mesure placés dans des glacières où ils sont conservés au frais à $4{ }^{\circ} \mathrm{C}$ au moyen d'accumulateurs de froid. Au laboratoire les échantillons d'eau sont transférés au réfrigérateur à $4{ }^{\circ} \mathrm{C}$ tandis que les sédiments sont conservés au congélateur à $-18{ }^{\circ} \mathrm{C}$ jusqu'à l'étape d'analyse 
qui intervient au plus tard dans les 72 heures qui suivent les prélèvements.

\section{Extractions des échantillons}

Les échantillons d'eau ne subissent aucune extraction avant le dosage des pesticides. Par contre les sédiments sont soumis à la méthode Quechers (Anastassiades et al., 2003). En effet, dans un tube à centrifuger de $50 \mathrm{~mL}$ on place $10 \mathrm{~g}$ d'échantillon de sédiment sec auquel on ajoute $10 \mathrm{~g}$ d'eau déionisée et $10 \mathrm{ml}$ d'acétonitrile. A l'issue d'une minute d'agitation sur agitateur Votex, le $\mathrm{pH} d u$ mélange est ajusté à 5-5,5 par ajout de $10 \mu \mathrm{L}$ de solution d'acide formique dans l'acétonitrile (à 5\%). Après une minute d'agitation, le mélange est centrifugé pendant $5 \mathrm{mn}$ à 4000 tours $/ \mathrm{mn}$. On récupère le surnageant qui est mis à congeler pour éliminer les matières grasses éventuelles. L'étape d'extraction des pesticides est amorcée par ajout de $25 \mathrm{mg} / \mathrm{ml}$ d'éthylenediamine-N-propyl (PSA) et 150 $\mathrm{mg} / \mathrm{ml}$ de $\mathrm{MgSO}_{4}$ : tout en favorisant l'extraction des pesticides tant polaires qu'apolaires grâce à la chaîne carbonée relativement longue et polaire du PSA, cet apport permet de désaliniser le mélange en chélatant les contres ions des sels. A l'issue d'une dernière opération de centrifugation, le surnageant est récupéré et utilisé pour les différents tests de dosage des pesticides par la technique ELISA ou toutes autres techniques analytiques (GC-ECD/SM ou HPLCUV/SM...).

\section{La technique ELISA}

L'ELISA est une technique d'immunoanalyse basée sur les interactions de nature immunologique (relations antigène-anticorps) pour le dosage de différents paramètres dont les pesticides. En technique ELISA, il existe deux méthodes à savoir la méthode en phase liquide et la méthode en phase solide. Pour le présent travail l'ELISA en phase liquide a été utilisé où les molécules d'immunoglobulines libres en solution ne sont fixées qu'à des particules magnétiques qui permettent de les coller aux fonds des tubes à essais à l'aide d'un rack magnétique lors des changements de milieu réactionnel pour la poursuite de l'expérience comme illustré par la Figure 3. L'échantillon à analyser, l'analogue structural de l'antigène marqué à l'enzyme et l'anticorps lié à des particules magnétiques se trouvent en présence dans le milieu réactionnel. Une compétition s'engage alors entre l'antigène naturel recherché et son analogue structural (haptène) vis-à-vis de l'anticorps. Des complexes binaires (anticorps-antigènes) et ternaires (anticorps-antigènes-enzymes) sont alors formés : c'est la réaction anticorpsantigène. Ces complexes binaires et/ou ternaires formés sont décantés à l'aide d'un séparateur magnétique et le surnageant est jeté. Deux séries de trois lavages successifs à l'aide d'une solution de Tween 20 (1x) permettent d'éliminer les composés indésirables et de minimiser les bruits de fond.

Pour la mesure de la concentration de l'antigène naturel recherché (pesticides), la proportion d'analogue structural à l'antigène est évaluée en servant à l'enzyme qui est la peroxydase, un substrat chromophorique incolore (le 3,3',5,5'-tétraméthylbenzidine dissous dans une base organique). Sa digestion développe une coloration (bleue) qui passe au jaune après ajout de la solution d'arrêt qui est une solution aqueuse d'acide sulfurique $0,5 \%$. L'intensité de la coloration est inversement proportionnelle à la concentration de l'antigène recherché dans l'échantillon testé. La coloration est mesurée par spectrophotométrie d'absorption moléculaire à une longueur d'onde de $450 \mathrm{~nm}$ avec un spectrophomètre de type $\mathrm{HACH}$ DR 2500 .

\section{Dosage des résidus de pesticides recherchés}

Les résidus de pesticides sont directement dosés dans les échantillons d'eau par la méthode ELISA, à l'exception du glyphosate dont le dosage passe préalablement par une technique de dérivation qui permet de transformer le glyphosate en Acide aminométhylphosphonique (AMPA) un 
dérivé aisément détectable par la technique ELISA utilisée.

Dans le souci de rester dans les domaines de linéarité de la réponse du détecteur colorimétrique utilisé, chaque échantillon a été analysé en procédant à des dilutions suivantes: échantillon non dilué, dilution $1 / 2,1 / 10,1 / 50$ et $1 / 100$. Chaque dilution est répétée trois fois. L'eau milliQ a servi de solvant de dilution pour l'atrazine et le glyphosate. Les pyréthrinoïdes sont dilués au méthanol pur (Merck).

Les dosages des échantillons naturels sont effectués concomitamment avec ceux du blanc et des molécules de référence de concentrations connues contenues dans les kits fournis par le fabricant, la société américaine ABRAXIS (référence PA18974).

Les gammes de détection de la méthode ELISA varient d'une molécule à une autre: $0,1-5 \mathrm{ppb}$ pour le glyphosate, $0,05-5 \mathrm{ppb}$ pour l'atrazine, $0,75-15 \mathrm{ppb}$ pour les pyréthrinoïdes.

\section{Validation de la Méthode d'extraction des sédiments}

Pour la validation de la méthode d'extraction une matrice de sédiment marin côtier de la plage de fidjrossè à Cotonou de granulométrie fine $<0,5 \mu \mathrm{m}$ a été désalée par trois séjours successifs de 24 dans de l'eau désionisée. Et un séjour de $24 \mathrm{~h}$ dans de l'eau milliQ suivi d'un séchage de $48 \mathrm{~h}$ à $625{ }^{\circ} \mathrm{C}$ dans un four carbolite pour éliminer toute la matière organique. Après séchage et extraction par la méthode Quechers, le sédiment marin utilisé a été testé négatif par plusieurs expérimentateurs du laboratoire. Ce sédiment marin ainsi testé est conservé dans du papier aluminium à $-18{ }^{\circ} \mathrm{C}$ pour les tests ultérieurement. Après dégèle à la température ambiante, 3 lots de $50 \pm 0,01 \mathrm{~g}$ de sédiment ont été contaminés à 0,$75 ; 3$ et $5 \mathrm{ng} / \mathrm{g}$ par molécule étudiée avec un mélange de solutions de référence constitué respectivement de Permétrine, de glyphosate et d'atrazine de concentration de $10 \mu \mathrm{g} / \mathrm{mL}$ pour chaque matière active dans un mélange méthanol-eau. Tous les réactifs ont été obtenus du Dr Gary Hinshaw de la société ABRAXIS (USA). Chacun des trois échantillons de sédiment contaminé est bien malaxé à l'aide d'une baguette de verre dans un bécher propre et placé sous agitation pendant 72 heures à $30{ }^{\circ} \mathrm{C}$ à l'abri de la lumière. Après refroidissement, 5 fractions de $10 \pm 0,01 \mathrm{~g}$ ont été effectuées par lot de sédiment contaminé et placées au congélateur à $-18{ }^{\circ} \mathrm{C}$. Pendant 5 jours successifs, une fraction de chacun des niveaux de contamination sus-cités (03) est dégelée, extraite par la méthode QUECHERS et dosée par la méthode ELISA en phase liquide. Pour chaque extrait les dilutions : échantillon non dilué, dilution $1 / 2$ et $1 / 10$ ont été effectuées. Chaque dilution est répétée trois fois. L'expérience est reprise six fois par jour pour chacune des molécules recherchées (Permétrine, glyphosate et atrazine). Soit un total de 810 essais (3 niveaux de contamination $\mathrm{x} \quad 3$ dilutions $\mathrm{x} \quad 3$ répétition/dilution $\quad x \quad 6 \quad$ essais/j $\quad x \quad 5 j) \quad$ par molécule de pesticide suivie. Ainsi les taux de récupération moyens $(\mathrm{R})$ et les coefficients de variation (\% CV qui exprime la distribution des valeurs enregistrées par rapport à la moyenne, afin de voir le degré d'homogénéité des données obtenues) sont calculés pour estimer la reproductibilité (Re) de l'expérience par les formules suivantes :

$$
\mathrm{Ri}=\frac{\mathrm{Qi}}{\mathrm{Qd}} \times 100
$$

Avec $\mathrm{Ri}=$ taux de récupération pour chaque niveau de contamination, $\mathrm{Qi}=$ valeur moyenne corrigée de la quantité de pesticides récupérée pour chaque niveau de contamination (en multipliant les moyennes au niveau de chaque dilution par le facteur de dilution et enfin la somme de moyenne ainsi corrigée divisée par 3) et Qd la quantité initiale de pesticides dans la fraction analysée de sédiment dopé. Qi et Qd sont rapportés à l'unité de masse de sédiment analysé.

$$
\mathrm{R}=\frac{\sum \mathrm{Ri}}{3}
$$


Avec i allant de 1 à 3 (trois niveaux de contamination).

$$
\operatorname{Re} \text { ou } \% \mathrm{CV}=\frac{\sigma \mathrm{i}}{\chi_{\mathrm{i}}} \times 100
$$

Avec $\sigma i$ l'écart type et $\chi \mathrm{i}$ la moyenne arithmétique des valeurs analytiques obtenues pour chaque niveau de contamination.

\section{Validation de la méthode d'analyse}

En dehors des solutions standards, un contrôle interne est effectué dans les mêmes conditions que les échantillons à doser. Il s'agit d'une solution de référence de glyphosate à $10 \mu \mathrm{g} / \mathrm{mL}$. La concentration de cette solution est indiquée sur le contenant et fournie avec les kits. Cette manière de procéder permet d'évaluer l'efficacité de la méthode et la correction des résultats d'analyse.

Efficacité $=\frac{\text { Concentration moyenne corrigée }}{\begin{array}{l}\text { Concentration indiquée par le } \\ \text { fabriquant }\end{array}} \times 100$

Le facteur de correction des résultats est donné par l'inverse de l'efficacité multiplié par cent (100).

$$
\text { Re ou } \% \mathrm{CV}=\frac{\sum \mathrm{Ri}}{\mathrm{Qd}} \times 100
$$

\section{Analyse statistique}

Les données ont été analysées à partir des valeurs du coefficient de variation (\%CV) obtenues à l'aide du logiciel XLstat 2009. Le coefficient de variation (en \%) permet de montrer le degré d'homogénéité des mesures. Il est obtenu par l'expression ci-dessus mentionnée. Selon Zogo et al. (2010) :

- $\quad$ si $\mathrm{CV}<2 \%$, les mesures sont très homogène et l'expérience reproductible ;

- $\quad$ si $2 \%<\mathrm{CV}<30 \%$, les mesures sont homogènes ;

- si CV > 30\%, les mesures sont hétérogènes donc l'expérience pour notre cas non reproductible.

\section{RESULTATS \\ Validation}

Le Tableau 3 présente les résultats d'analyse de la matrice de sédiment témoin: Les moyennes de pourcentage de récupération varient de $95 \%$ à $105 \%$ pour la permétrine, le glyphosate et l'atrazine contre 98 à 106 prévus par le fabricant des Kits. Ce qui montre la fiabilité des résultats de la méthode de même que sa reproductibilité en ce sens que les $\% \mathrm{CV}$ sont compris entre $1,94 \%$ et $30 \%$. Ce qui à permis l'utilisation de cette méthode EIISA pour des mesures en milieu naturel.

Teneur des résidus de pesticides dans les systèmes naturels

Les Figures 4,5 et 6 donnent l'évolution des taux de triazine, d'aminophosphate et de pyréthrinoïdes dans l'eau et dans les sédiments de la rivière Agbado en saisons sèche et pluvieuse.

\section{Concentrations des résidus de pesticides dans l'eau et les sédiments}

En saison sèche aucun résidu de pesticide recherché n'a été détecté dans l'eau. Par contre en saison pluvieuse (Figure 4), ils ont été trouvés dans l'eau de la rivière à des concentrations allant de 0,05 à 0,475 ppb pour les triazines, de 0,10 à $1,316 \mathrm{ppb}$ pour les aminophosphates et de 0,75 à 4,45 ppb pour les pyréthrinoïdes, ces derniers sont les plus abondants sur l'ensemble des sites. De façon générale, nos valeurs sont très faibles par rapport à celles de Ioannis et al. (2006) qui ont enregistré des valeurs allant jusqu'à 5900 ppb pour les triazines et 3000 ppb pour les pyréthrinoïdes dans les eaux de surface en Grèce. Des valeurs relativement basses que celles de la Grèce, mais toujours plus élevées que les nôtres qui sont de l'ordre de 100 à 1000 ppb pour les triazines ont été détectées dans les eaux de surfaces en France par Tisseau et al. (1999). Cet état de chose trouve son explication dans le fait que dans les pays africains au sud du Sahara, les exploitations agricoles sont de petites tailles et les techniques culturalles sont rudimentaires et moins polluantes, contrairement aux grosses 
exploitations industrialisées des pays du nord où les consommations en pesticides sont plus importantes avec des modes de pulvérisation à grandes échelles plus nuisibles à l'environnement.

Dans les sédiments, en saison sèche, les aminophosphates ont été détectés seulement sur le site 1 (Mèdétèkpo) à un taux de 0,39 ppb. En dehors d'eux seules les triazines ont été détectées sur tous les sites à un taux variant entre 0,22 et $0,97 \mathrm{ppb}$. Ces derniers résidus en saison pluvieuse tout comme les pyréthrinoïdes en saison sèche et pluvieuse n'ont pas été détectés dans les sédiments. Par contre en saison pluvieuse seuls les aminophosphates ont été détectés au niveau de trois sites sur quatre. Sa teneur moyenne varie sur ces sites de 6,8 à 8,62 ppb montrant sa forte prévalence en période de pluie. En moyenne les teneurs de pesticides sont inférieures aux limites admissibles au Bénin pour l'eau de boisson qui sont de $5 \mathrm{ppb}$ pour l'atrazine, $20 \mathrm{ppb}$ pour les pyréthrénoïdes et 280 ppb pour le glyphosate (Décret n²001094, 2001). Si ces concentrations restent faibles par rapport aux recommandations de l'Organisation Mondiale de la Santé (2 ppb pour l'atrazine et $9 \mathrm{ppm}$ pour le glyphosate), elles sont cependant parfois très élevées comparativement aux limites recommandées par l'Union Européenne et qui sont de 0,1 ppb pour chacun des pesticides ou leur produit de dégradation et $5 \mathrm{ppb}$ pour leur somme.

Tableau 1 : types et quantité de pesticides utilisés en agriculture au Bénin de 1999 à 2008.

\begin{tabular}{|c|c|c|c|c|}
\hline Campagnes & $\begin{array}{l}\text { Nature des } \\
\text { pesticides }\end{array}$ & $\begin{array}{c}\text { Noms } \\
\text { Commerciaux } \\
\end{array}$ & Matières actives & $\begin{array}{c}\text { Quantité } \\
\text { (litres) }\end{array}$ \\
\hline \multirow{4}{*}{$1999-2000$} & \multirow{4}{*}{ Insecticides } & Phaser & Endosulfan & \multirow{4}{*}{69456} \\
\hline & & Dursban B & Chlorpyriphos éthyl & \\
\hline & & Cotalm D & $\begin{array}{l}\text { Lambdacyhalothrine- } \\
\text { profenophos }\end{array}$ & \\
\hline & & Sherphos & Cyperméthrine-triazophos & \\
\hline \multirow{4}{*}{$2000-2001$} & \multirow{4}{*}{ Insecticides } & Phaser & Endosulfan & \multirow{4}{*}{45140} \\
\hline & & Dursban B & Chlorpyriphos éthyl & \\
\hline & & Cotalm D & $\begin{array}{l}\text { Lambdacyhalothrine- } \\
\text { profenophos }\end{array}$ & \\
\hline & & Sherphos & Cyperméthrine-triazophos & \\
\hline \multirow{4}{*}{ 2001-2002 } & \multirow{4}{*}{ Insecticides } & Phaser & Endosulfan & \multirow{4}{*}{17544} \\
\hline & & Dursban B & Chlorpyriphos éthyl & \\
\hline & & Cotalm D & $\begin{array}{l}\text { Lambdacyhalothrine- } \\
\text { profenophos }\end{array}$ & \\
\hline & & Sherphos & Cyperméthrine-triazophos & \\
\hline \multirow{4}{*}{$2002-2003$} & \multirow{4}{*}{ Insecticides } & Phaser & Endosulfan & \multirow{4}{*}{51684} \\
\hline & & Dursban B & Chlorpyriphos éthyl & \\
\hline & & Cotalm D & $\begin{array}{l}\text { Lambdacyhalothrine- } \\
\text { profenophos }\end{array}$ & \\
\hline & & Sherphos & Cyperméthrine-triazophos & \\
\hline \multirow{8}{*}{$2003-2004$} & \multirow{6}{*}{ Insecticides } & Thionex & Endosulfan & \multirow{6}{*}{15609} \\
\hline & & Lambdacal & $\begin{array}{l}\text { Lambdacyhalothrine- } \\
\text { profenophos }\end{array}$ & \\
\hline & & Cotalm & $\begin{array}{l}\text { Lambdacyhalothrine- } \\
\text { profenophos }\end{array}$ & \\
\hline & & Conquest & Cyperméthrine-acetamipride & \\
\hline & & Curacron & Profenophos & \\
\hline & & Sherphos & Cyperméthrine-triazophos & \\
\hline & \multirow{2}{*}{ Herbicides } & Callifor $\mathrm{G}$ & $\begin{array}{l}\text { Prométrine Fluométuron } \\
\text { Glyphosate }\end{array}$ & \multirow{2}{*}{269} \\
\hline & & Primagramme & Atrazine ; Metolachlore & \\
\hline
\end{tabular}


M. A. N. GBAGUIDI et al. / Int. J. Biol. Chem. Sci. 5(4): 1476-1490, 2011

\begin{tabular}{|c|c|c|c|c|}
\hline \multirow{6}{*}{$2005-2006$} & \multirow{3}{*}{ Insecticides } & Thionex & Endosulfan & \multirow{3}{*}{36772} \\
\hline & & Lambdacal & $\begin{array}{l}\text { Lambdacyhalothrine- } \\
\text { profenophos }\end{array}$ & \\
\hline & & Conquest & Cyperméthrine-acetamipride & \\
\hline & \multirow{3}{*}{ Herbicides } & Callifor G & $\begin{array}{l}\text { Prométrine Fluométuron } \\
\text { Glyphosate }\end{array}$ & \multirow{3}{*}{1063} \\
\hline & & Primagramme & Atrazine ; Metolachlore & \\
\hline & & Garil & Triclopyr ; Propanil & \\
\hline \multirow{8}{*}{ 2006-2007 } & \multirow{4}{*}{ Insecticides } & Thionex & Endosulfan & \multirow{4}{*}{31401} \\
\hline & & Sherphos & Cyperméthrine-triazophos & \\
\hline & & Lambdacal & $\begin{array}{l}\text { Lambdacyhalothrine- } \\
\text { profenophos }\end{array}$ & \\
\hline & & Conquest & Cyperméthrine-acetamipride & \\
\hline & \multirow{4}{*}{ Herbicides } & Callifor G & $\begin{array}{l}\text { Prométrine Fluométuron } \\
\text { Glyphosate }\end{array}$ & \multirow{4}{*}{848} \\
\hline & & Primagramme & Atrazine ; Metolachlore & \\
\hline & & Garil & Triclopyr ; Propanil & \\
\hline & & Galant super & Haloxyfop méthyle ester & \\
\hline \multirow{7}{*}{ 2007-2008 } & \multirow{3}{*}{ Insecticides } & Cotofan & Endosulfan & \multirow{3}{*}{40642} \\
\hline & & Cyflutralm & Cyfluthrine-profenofos & \\
\hline & & Capt & Cyperméthrine-acetamipride & \\
\hline & \multirow{4}{*}{ Herbicides } & Callifor G & $\begin{array}{l}\text { Prométrine Fluométuron } \\
\text { Glyphosate }\end{array}$ & \multirow{4}{*}{2136} \\
\hline & & Primagramme & Atrazine ; Metolachlore & \\
\hline & & Garil & Triclopyr ; Propanil & \\
\hline & & Kalach & Glyphosate & \\
\hline
\end{tabular}

Tableau 2 : Sites d'échantillonnage avec leurs coordonnées géographiques et les périodes de prélèvement.

\begin{tabular}{|c|c|c|c|c|}
\hline \multirow{2}{*}{$\begin{array}{l}\text { Nom des } \\
\text { d'échantillonnage }\end{array}$} & \multicolumn{2}{|c|}{ Coordonnées géographiques } & \multicolumn{2}{|c|}{ Périodes de prélèvement } \\
\hline & $\mathrm{X}$ & $\mathrm{Y}$ & Saison sèche & $\begin{array}{l}\text { Saison } \\
\text { pluvieuse }\end{array}$ \\
\hline Médétokpo & $02^{\circ} 01^{\prime} 47,64^{\prime \prime}$ & $08^{\circ} 05^{\prime} 20,32^{\prime}$, & \multirow{4}{*}{$\begin{array}{l}1^{\text {ère }} \text { campagne } \\
\text { du } 09 \text { au } \\
14 / 10 / 2006 \\
\text { (début saison } \\
\text { sèche) } \\
2^{\text {ème campagne }} \\
\text { du } 14 \text { au } \\
21 / 12 / 2006 \text { (fin } \\
\text { saison sèche) }\end{array}$} & \multirow{3}{*}{$\begin{array}{l}1^{\text {ère }} \text { campagne } \\
\text { du } 04 \mathrm{au} \\
\text { 09/05/2007(déb } \\
\text { ut saison } \\
\text { pluvieuse) }\end{array}$} \\
\hline Barrage de la SONEB & $02^{\circ} 00^{\prime} 20,58^{\prime \prime}$ & $07^{\circ} 57^{\prime} 19,46^{\prime}$, & & \\
\hline $\begin{array}{llll}\text { Station de } & \text { traitement de } \\
\text { SONEB } & & & \\
\end{array}$ & $01^{\circ} 59^{\prime} 33,70^{\prime \prime}$ & $07^{\circ} 53^{\prime} 35,90^{\prime \prime}$ & & \\
\hline Sous le grand pont de Gobada & $01^{\circ} 58^{\prime} 40,48^{\prime \prime}$ & $07^{\circ} 46^{\prime} 08,98^{\prime \prime}$ & & $\begin{array}{l}2^{\text {ème }} \text { campagne } \\
\text { du } 10 \text { au } \\
17 / 09 / 2007 \text { (fin } \\
\text { saison } \\
\text { pluvieuse) }\end{array}$ \\
\hline
\end{tabular}


M. A. N. GBAGUIDI et al. / Int. J. Biol. Chem. Sci. 5(4): 1476-1490, 2011

Tableau 3 : Résultats de validation de la méthode d'extraction et du test ELISA en phase liquide.

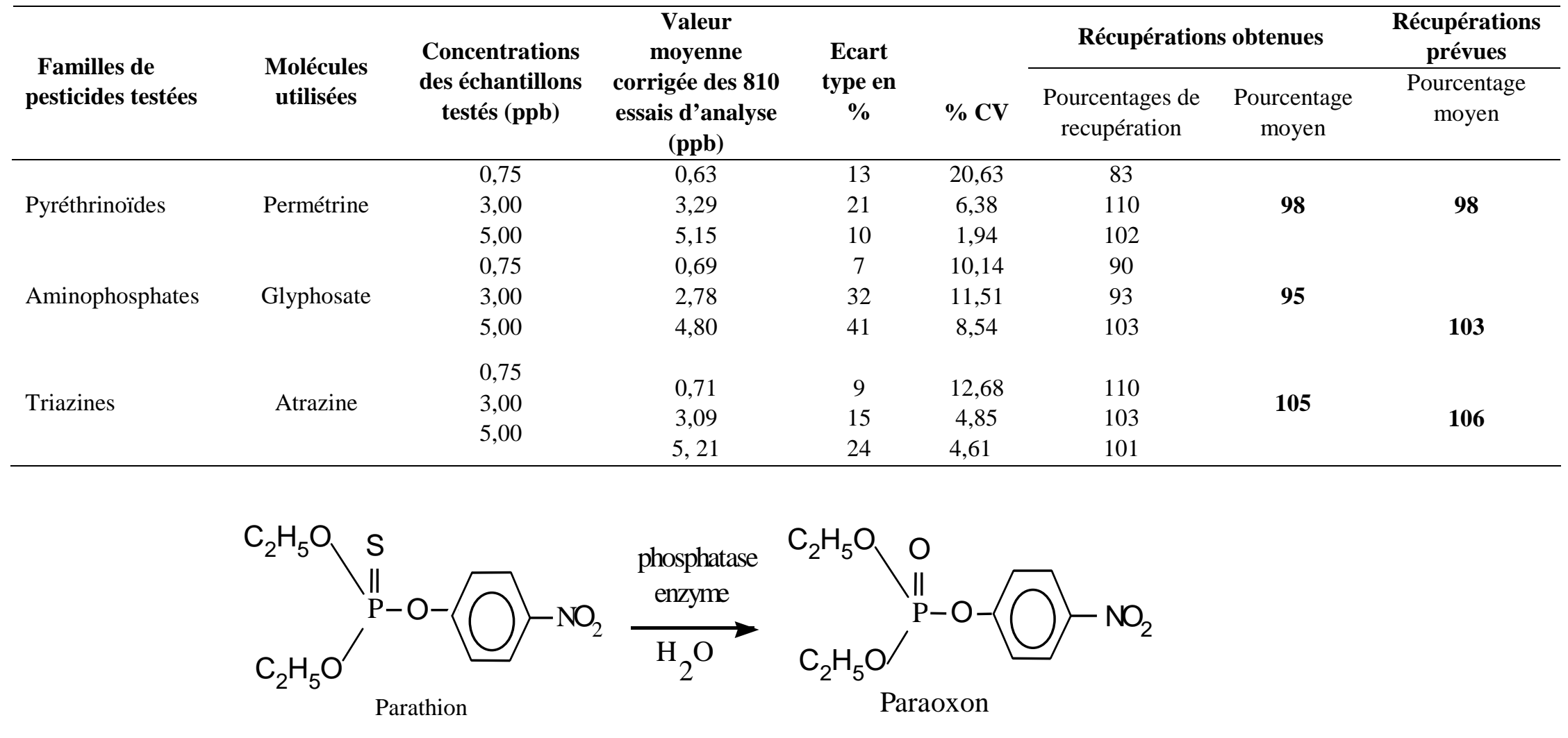

Figure 1 : Dégradation du Parathion en Paraxon. 


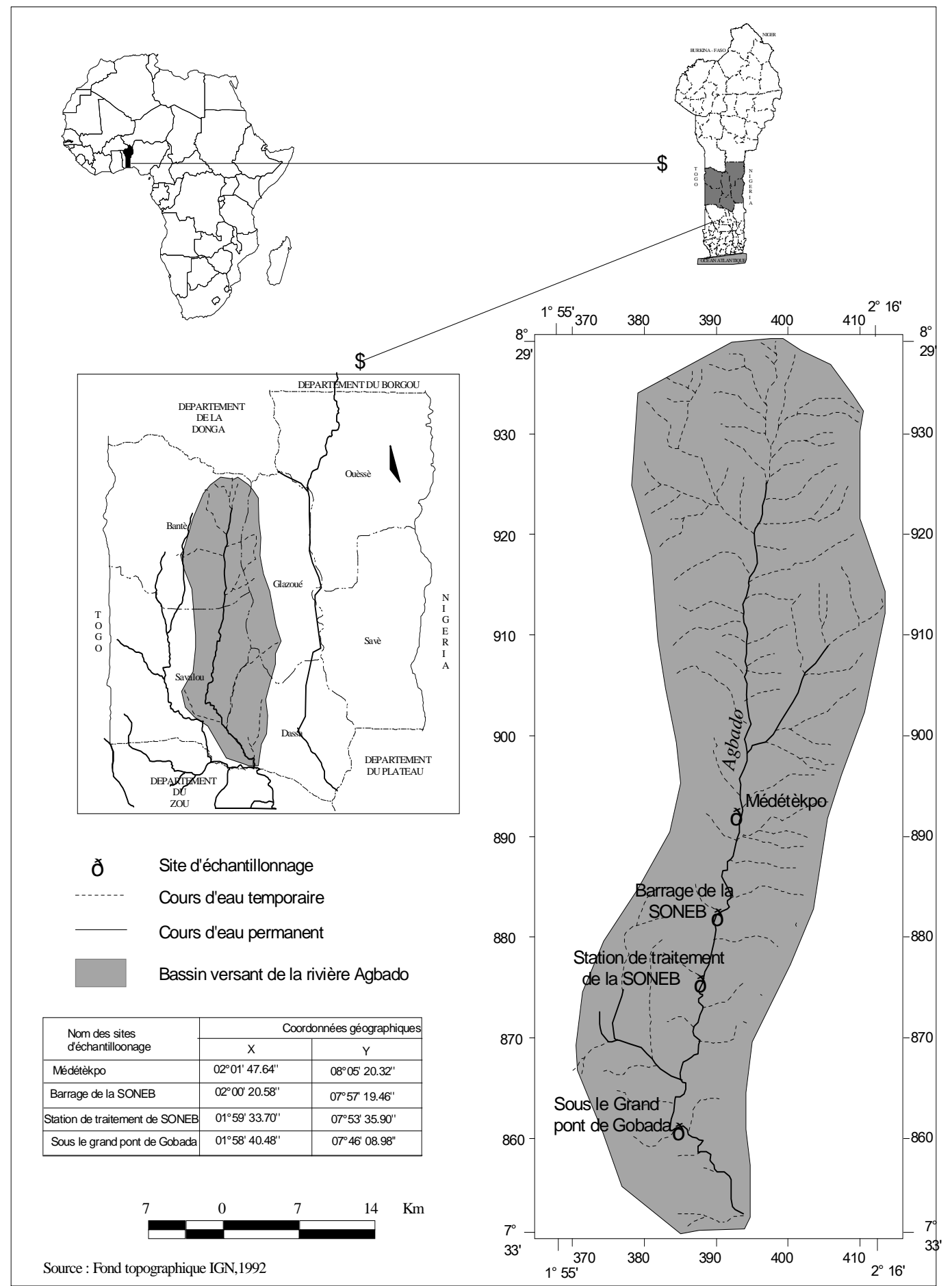

Figure 2 : Cartes de situation de la zone d'étude comportant les sites et leurs coordonnées géographiques. 


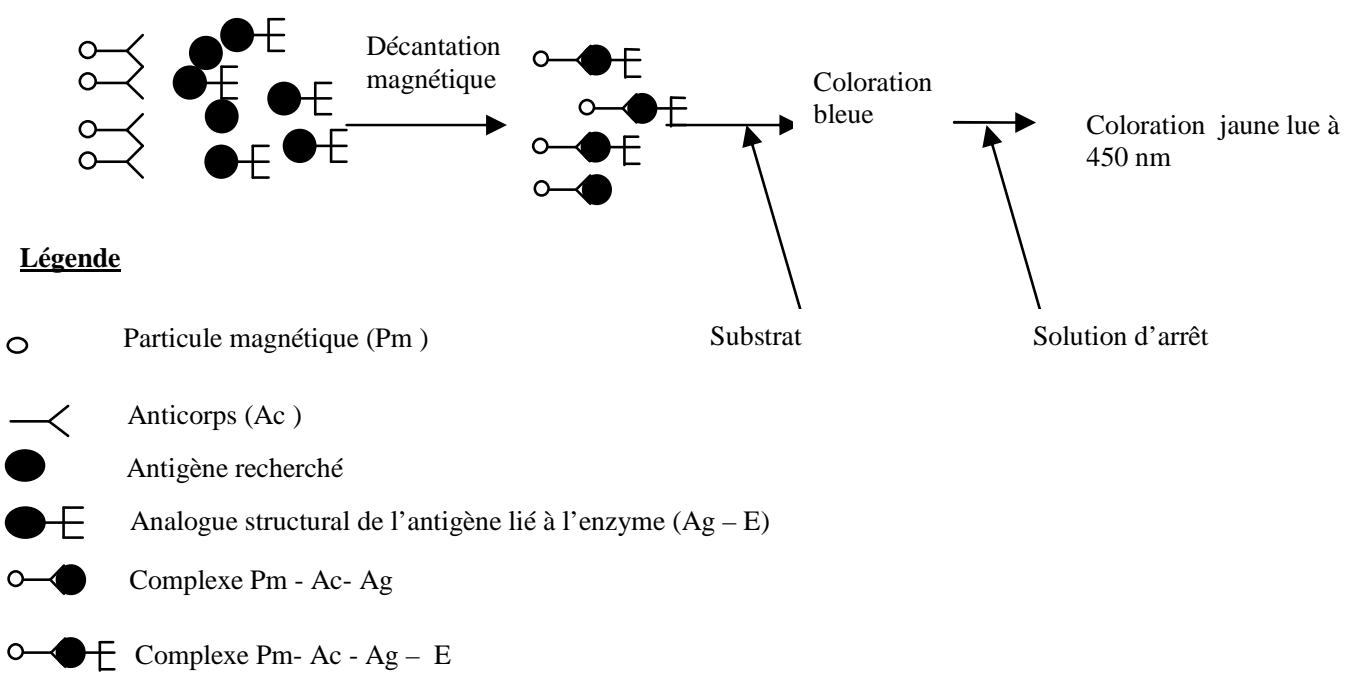

Figure 3 : Illustration des étapes de la technique ELISA en phase liquide

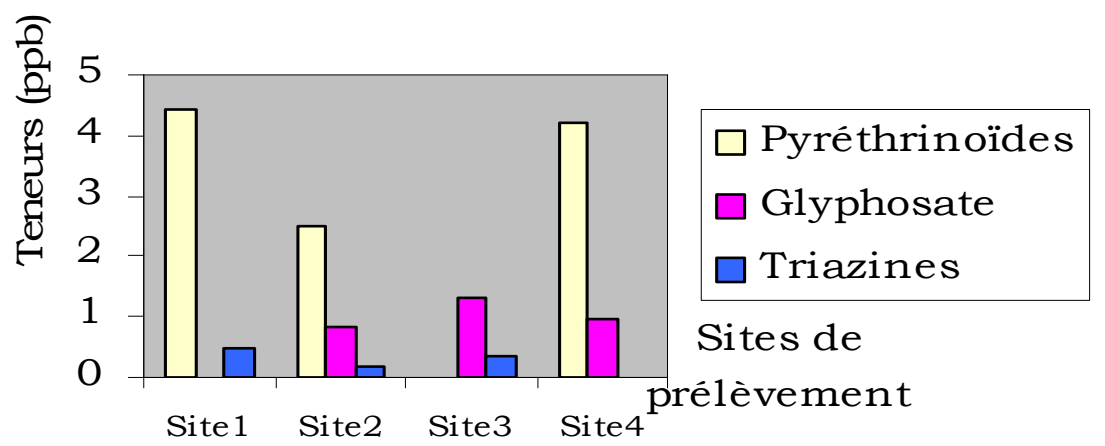

Figure 4: Teneurs des résidus de pesticides dans l'eau de la rivière Agbado en saison pluvieuse.

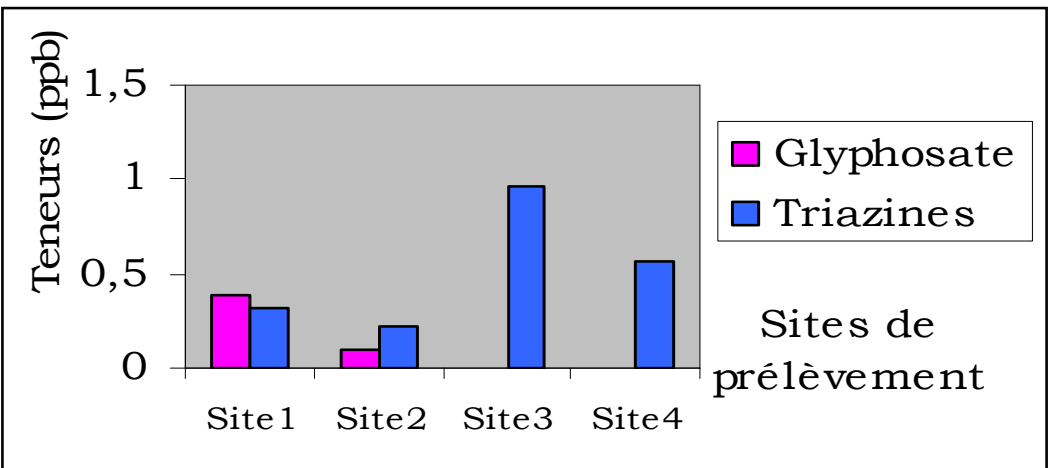

Figure 5 : Teneurs des résidus de pesticides dans les sédiments de la rivière Agbado en saison sèche. 


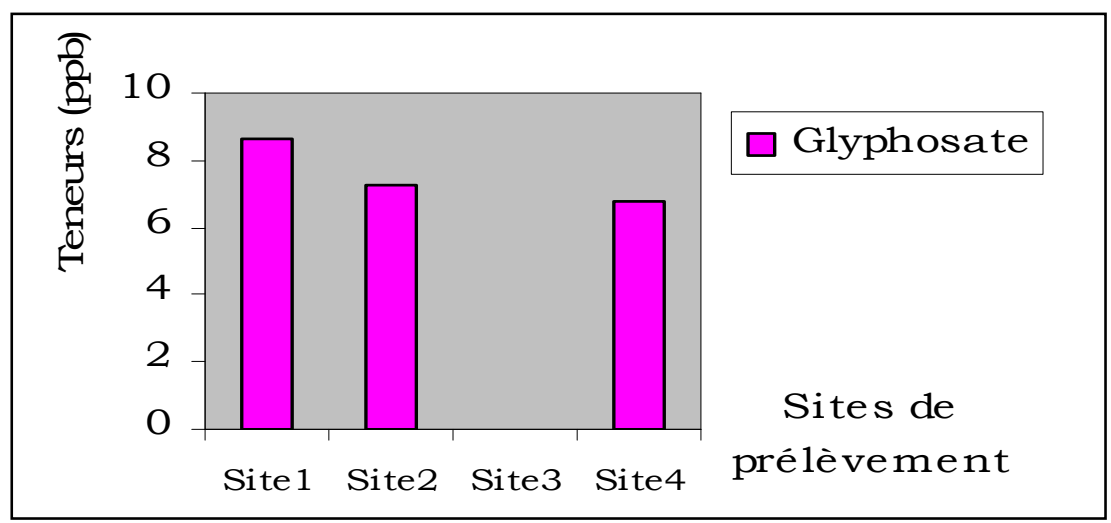

Figure 6: Teneurs des résidus de pesticides dans les sédiments de la rivière Aghado en saison nlıvieuse.

\section{DISCUSSION}

\section{Origine des résidus de pesticides}

Les pyréthrinoïdes sont les plus abondants dans l'eau sauf au niveau du site 3 (station de traitement d'eau de la SONEB) où leur présence n'a pas été perceptible. Les types de pyréthrinoïdes importés au Bénin pour la lutte antivectorielle sont principalement la cyperméthrine, la cyflutrine et le lambdacyhalothrine (CNAC, 2007). Ils sont liposolubles (avec un coefficient de partage octanol/eau $\mathrm{K}_{\mathrm{ow}}$ compris entre 6 et 6,3 , neutres, très peu volatils et instables chimiquement (temps de demi-vie ou $\mathrm{DT}_{50}$ compris entre 5 jours et 13 semaines dans le sol) (Fournier, 1988). Cette instabilité chimique des pyréthrinoïdes dans la nature permet de justifier plus les faibles taux enregistrés plutôt que leur utilisation raisonnée. Normalement l'activité insecticide plus forte de ces produits chimiques devrait permettre d'avoir recours à des doses relativement modestes; mais l'ignorance couplée à l'analphabétisme des agriculteurs les amènent à une utilisation abusive de ces pesticides de synthèse (Lafia, 1996). Même si leurs effets sur l'homme sont encore très mal connus, la pratique culturale a entrâné l'apparition des résistances tant au niveau des vecteurs cibles qu'au niveau des vecteurs non cibles tel que l'anophèle gambiae. Cet état de chose d'une part, complique aujourd'hui le choix d'un insecticide dans la lutte antivectorielle (LAV) contre le paludisme (Martin et al., 2007), et d'autre part explique le recours abusif, des agriculteurs aux pesticides organochlorés comme l'endosulfan et même le DDT au Bénin (Soclo et al., 2003; Elisabeth et al., 2006a et b).

Les aminophosphates sont la deuxième famille de pesticides les plus abondants étudiés dans les eaux où ils ont été retrouvés sur tous les sites à l'exception du site1 (Mèdétèkpo) en saison pluvieuse. Dans les sédiments, ils sont beaucoup plus abondants que les autres résidus de pesticides. Tous les échantillons de sédiments prélevés en saison pluvieuse sont contaminés. Par contre ils n'ont été détectés en saison sèche qu'au niveau des sites1 (Mèdétèkpo) et 2 (Barrage SONEB). Le glyphosate [N(phosphonométhyl) glycine] est le seul des aminophosphates utilisé en agriculture au Bénin. C'est un herbicide systémique postémergent ayant un spectre d'application large (Blackburn et Boutin, 2003; Couteux et Lejeune, 2003). C'est l'herbicide le plus utilisé actuellement au niveau mondial (FAO, 2004). Ainsi pour limiter la main d'œuvre pour le sarclage et disposer de temps pour d'autres 
activités génératrices de revenus complémentaire à ceux de l'agriculture comme la conduite de taxi moto "zémidjan », les jeunes agriculteurs abusent parfois de son utilisation. Or le glyphosate et son métabolite principal, l'AMPA (acide aminométhylphosphonique), sont très solubles dans l'eau (10.500 ppm) (Solomon et Thompson, 2003); par conséquent ils se retrouvent en solution aqueuse après lessivage des sols traités et peuvent poser des problèmes de contamination au niveau des eaux superficielles. Malgré sa forte solubilité aqueuse, son coefficient d'adsorption aux particules du sol Koc est de 21.699 (Couteux et Lejeune, 2003). Cette forte adsorption aux particules du sol pourrait expliquer la présence du glyphosate à une teneur très élevée dans les sédiments qu'il peut facilement atteindre par gravité en transitant par les particules en suspension dans l'eau. Il est très toxique pour les poissons, les alevins et les phytoplanctons principaux maillon de la chaîne trophique (Smith et Oehme, 1992).

S'agissant de la famille des triazines, c'est l'atrazine qui est utilisé. Le seul usage rapporté pour l'atrazine au Bénin est son action herbicide pour des usages agricoles (désherbage des champs de culture) (CNAC, 2007). Il est observé en saison pluvieuse sur tous les sites à l'exception de l'échantillon d'eau du site 4 (grand pont de Gobada). Quant aux sédiments, ils sont tous contaminés par l'atrazine en saison sèche. Cependant, les échantillons analysés ont révélé des taux inférieurs à la limite de 3 ppb admissible par l'Agence Américaine pour la Protection de l'Environnement (US-EPA) (Brignon et Gouzy, 2007). Les faibles taux de l'atrazine trouvés dans l'eau seraient liés à sa faible hydrosolubilité $\left(33\right.$ ppb à $20^{\circ} \mathrm{C}$ ) (Couteux et Lejeune, 2003). Selon les mêmes auteurs son coefficient d'adsorption (Koc) est aussi relativement faible variant de 38 à 170 , d'où probablement l'absence des triazines dans les sédiments de surface en saison pluvieuse.

\section{Conclusion}

La technique ELISA en phase liquide a permis de détecter et de quantifier les pesticides tels que les pyréthrinoïdes, le glyphosate et l'atrazine dans l'eau en saison pluvieuse. Dans les sédiments le glyphosate a été détecté tant en saison sèche qu'en saison pluvieuse. Par contre l'atrazine n'a été détecté dans les sédiments qu'en saison sèche. Toutes les concentrations enregistrées sont largement en dessous des normes béninoises et des limites admissibles par l'OMS pour l'eau de boisson.

Ces teneurs relativement faibles trouvent leur explication beaucoup plus dans le caractère moins rémanent des molécules recherchées que dans une utilisation raisonnée de ces dernières. Ainsi dans les pays moins avancés, la technique ELISA peut être une bonne alternative aux techniques sophistiquées et très onéreuses pour l'analyse des pesticides. La présence diffuse de pesticides dans les eaux et surtout des herbicides est une source de menaces à court, moyen et long terme pour l'homme et l'environnement si les tendances actuelles ne sont pas inversées. En effet les herbicides constituent des risques potentiels de destruction des phytoplanctons, nourritures de certains organismes qui, naturellement ne constituent pas un danger pour les cultures. Dépourvus de leur nourriture ils peuvent s'attaquer aux cultures et de ce fait, pourront constituer une nouvelle classe de nuisibles.

\section{REMERCIEMENTS}

Tous nos remerciements à l'endroit:

- De la Fondation international pour la science IFS et de l'Université d'Abomey-Calavi pour leurs soutient financier.

- Principalement du Dr Oman Cecilia, de Dr Ericksson Sune, de Edward Rumilia, du Dr Klustsé Amah et mes collègues pour nos franches collaborations.

\section{REFERENCES}

Anastassiades M, Lehotay S, Stajnbaher D, Schenck F. 2003. Fast and easy multiresidue method employing acetonitrile extraction/partitioning and "dispersive solid-phase extraction" for the determination of pesticide residues in produce. J. AOAC. Int., 86(2): 412-31. 
Banque mondiale. 2004. Reformes du secteur du coton: une analyse de la pauvreté et de l'impact social .Rapport No 29951-BJ BENIN, p. 80.

Blackburn LG, Boutin C. 2003. Subtle effects of herbicide use in the context of genetically modified crops: a case study with glyphosate (Roudup). Ecotoxicol., 12: 271-285.

Brignon JM, Gouzy A. 2007. Données Technico-économiques sur les Substances Chimiques en France, Atrazine. INERIS.

Celeste ML, Da-Silva IMN. 2006. Evalution of Organochlorine pesticides in serum from students in Coimbra, Portugal: 1997-2001. Environ. Res., 102(3): 339351.

CNAC (Comité National d'Agrément et de Control des produits phytopharmaceutiques). 2007. Liste des produits phytopharmaceutiques sous autorisation provisoire de vente (APV) et agrément homologation (AH). Bénin, Porto-Novo, p.9.

Couteux A, Lejeune V. 2003. Index phytosanitaire ACTA 2003-39, Paris : Association de Coordination Technique Agricole.

Decret N²001- 094 du 20 février 2001 fixant les normes de qualité de l'eau potable en Republique du Bénin. p 21.

Elisabeth YAP, Philippe L, Michel B, Cornelis AMVG, Hyacinthe A, Simon A, Bert VH, Kees S, Nico MVS. 2006a. Contamination of fish organochlorine pesticide residues in the Ouémé River catchment in the Republic of Bénin. Environnement Int., 32: 594-5999.

Elisabeth YAP, Michel B, Cornelis AMVG, Hyacinthe A, Philippe L, Simon A, Bert VH, Kees S, Nico MV S. 2006b. Organochlorine and organophosphorous pesticide residues in the Ouémé River catchment in the Republic of Bénin. Environnement Int., 32: 616-623.

FAO. 2004. FAO specifications and evaluations for agricultural pesticides. $\mathrm{p}$ 25 .
Fournier J. 1988. Chimie des Pesticides (1ère édition). Edition des trois Moutiers.

Garey AF, Rafael MC, George JS. 2010. Influence of flow concentration on parameter and prediction uncertainty of pesticide trapping by vegetative filter strips. Journal of Hydrology, 384: 164173.

Helena T, Paula P, Margarida A, Margarida O, Estela PM, Duarte NV. 2004. Pesticide intoxication in the centre of Portugal: three years analysis. Forensic Science International, 143: 199-204.

Hock B, Giersch T, Kramer K, Pullen S. 1994. Toxicity assessment and on-line monitoring: Immunoassays Environ. Toxicol. Water qual., 9: 243-262.

INSAE. 2002. Troisième Recensement Général de la Population et de l'Habitat (RGPH) au Bénin.

Ioannis KK, Dimitra GH, Triantafyllos AA. 2006. The status of pesticide pollution in surface waters (rivers and lakes) of Greece. Part I. Review on occurrence and levels. Environ. Pollu., 141: 555-570.

Katrijn MAH, Piet S, Peter AV. 2007. Monitoring and modelling pesticide fate in surface water at the catchment scale. Ecological Modelling, 209: 53-64.

Lafia E. 1996. Facteurs de risque et taux d'acéthycholinestérase chez les utilisateurs des pesticides dans la SousPréfecture de Banikoara. Thèse de Doctorat en Médecine, FSS/UNB Bénin, p. 93.

Marc J. 2004. Effets toxiques d'herbicides à base de glyphosate sur la régulation du cycle cellulaire et le développement précoce en utilisant l'embryon d'oursin. Thèse de doctorat de l'Université de Rennes 1, N³034, p.164.

Marina L, Inmaculada L-F, Miguel RB, Clemente AG, Julia BM, Oscar PM, Ricardo G, Susana B, Mariano EC. 2010. Association between organophosphate pesticides exposure and thyroid hormones floriculture workers. Toxicology and Applied Pharmacology, 243: 19-26. 
Martin T, Akogbéto M, Hougard JM, Rowl M, Corbel V, N'Guessan R, Brengues C, Chandre F, Djogbenou L. 2007. Multiple insecticide resistance mechanisms in Anopheles gambiae and Culex quinquefasciatus from Benin, West Africa. Acta Tropica, 101: 207-216.

Scheyer A. 2004. Développement d'une méthode d'analyse par CPG/MS/MS de 27 pesticides identifiés dans les phases gazeuse, particulaire et liquide de l'atmosphère. Application à l'étude des variations spatio-temporelles des concentrations dans l'air et dans les eaux de pluie. Thèse de doctorat, Université Louis Pasteur de Strasbourg, Alsace, p.273.

Smith EA, Oehme FW. 1992. The biological activity of glyphosate to plants and animals: a literature review. Vet. Hum. Toxicol., 34: 531-543.

SOCLO HH. 2003. Etude de l'impact de l'utilisation des engrais chimiques et des pesticides par les populations riveraines sur les écosystèmes (eaux de surfaces, substrats des réserves de faune) dans les complexes des aires protégées de la Pendjari et du W. CENAGREF 2003, p. 135.

Solomon KR, Thompson DG. 2003. Ecologicol risk assessment for aquatiquc organisms from over-water uses of glyphosate. J. Toxicol. Environ. Health B Crit. Rev., 289-324.

Tisseau MA, Fauchon N, Cavard J, Vandevelde T. 1999. Pesticide contamination of water resources: a case study the rivers in the Paris region. Water Sci. and Technol., 34(7-8): 147-152.

Traore SK, Dembele A, Mamadou K, Mambo V, Lafrance P, Bekro YA, Houenou P. 2008. Contrôle des pesticides organochlorés dans le lait et produits laitiers : Bioaccumulation et risques d'exposition. Afrique Sci., 04(1): 87 - 98.

Zogo D, Bawa LM, Soclo HH, Atchekpe D. 2010. Elimination de la couleur et de la turbidité d'une eau de surface par coagulation-floculation au sulfate d'aluminium: cas de la retenue d'eau de l'Okpara en République du Bénin. Int. J. Biol. Chem. Sci., 4(5): 1667-1675. 